BUHEP-95-21

\title{
Limits on Pseudoscalar Bosons From Rare $Z$ Decays at LEP
}

\author{
Gautam Rupak国 and Elizabeth H. Simmons \\ Department of Physics \\ Boston University \\ 590 Commonwealth Avenue \\ Boston, MA 02215
}

\begin{abstract}
Current LEP data on rare $Z$ decays constrain nonminimal technicolor models that contain light neutral pseudo-Nambu-Goldstone bosons $\left(P^{a}\right)$. We discuss the production and decay of such particles, and show how LEP data on $\gamma \gamma \gamma, \gamma+E$ and $\gamma+$ hadrons constrain the size of the technicolor gauge group and the strength of the $Z \gamma P^{a}$ coupling. The limits are then applied to several specific technicolor scenarios.
\end{abstract}

$7-95$

1 E-mail: grupak@buphy.bu.edu. Current address: Physics Department, University of Washington, Seattle WA 98195

2 E-mail: simmons@bu.edu 


\section{Introduction}

Data from experiments at the $\mathrm{Z}$ pole provide valuable information about models of electroweak symmetry breaking. In particular, LEP now probes rare $\mathrm{Z}$ decays with branching ratios at the $10^{-5}-10^{-6}$ level. This allows the possibility of detecting non-standard particles that couple very weakly to the $Z$.

Many non-minimal technicolor models include electrically neutral pseudo-NambuGoldstone bosons that couple anomalously to the photon and $Z$. These non-standard pseudoscalar particles $\left(P^{a}\right)$ can be produced at LEP through the process $Z \rightarrow \gamma P^{a}$ [1]. If the pseudoscalar's mass is less than about $65 \mathrm{GeV}$, the branching fraction can be of order $10^{-5}$. A dramatic feature of these rare $Z$ decays is the fact that the photon energy is uniquely fixed by the scalar's mass. Previous investigations [2] [3] of the production and decay modes of these neutral pseudoscalars have shown that all decays yield distinctive signatures for which the LEP experiments can search. Among the most interesting are events with three final state photons, because neither composite nor supersymmetric models should yield a visible signal in this channel [2].

This paper evaluates the limits that LEP data on rare $Z$ decays [4] [5] [6] [7] place on non-minimal technicolor models. We begin by discussing the rate of $P^{a}$ production and the available decay modes. Next, we explore the kinematics of the final states for which experiments can search. We study the limits on each final state and express them in terms of bounds on the size of the technicolor gauge group and the strength of the $Z \gamma P^{a}$ coupling. Finally, we apply our bounds to several specific technicolor scenarios.

\section{Production and Decay of $P^{a}$}

At $\sqrt{s}=M_{Z}$, the primary3 production mode for a neutral pseudo-Nambu-Goldstone boson (PNGB) of mass less than $M_{Z}$ is the two-body process $Z \rightarrow \gamma P^{a}$. For technifermions in the fundamental representation of technicolor, a PNGB couples to a pair of gauge bosons $G_{1}, G_{2}$ with charges $g_{1}, g_{2}$, momenta $k_{1}, k_{2}$ and polarizations $\epsilon_{1}, \epsilon_{2}$ just as a pion couples to a pair of photons [8]:

$$
N_{T C} \mathcal{A}_{G_{1} G_{2}} \frac{g_{1} g_{2}}{2 \pi^{2} f} \epsilon_{\mu \nu \lambda \sigma} k_{1}^{\mu} k_{2}^{\nu} \epsilon_{1}^{\lambda} \epsilon_{2}^{\sigma} .
$$

Here $\mathcal{A}_{G_{1} G_{2}}$ is the anomaly factor for the axial current $T^{a}$ associated with $P^{a}$

$$
4 \mathcal{A}_{G_{1} G_{2}}=\operatorname{Tr}\left[T^{a}\left(T^{1} T^{2}+T^{2} T^{1}\right)_{L}\right]+\operatorname{Tr}\left[T^{a}\left(T^{1} T^{2}+T^{2} T^{1}\right)_{R}\right]
$$

3 The sub-dominant processes $Z \rightarrow Z^{*} P^{a} \rightarrow f \bar{f} P^{a}$ and $Z \rightarrow b \bar{b} \rightarrow b \bar{b} P^{a}$ are discussed in [2] [3]. 
$T^{1}$ and $T^{2}$ are the generators associated with the gauge bosons, $L$ and $R$ refer to leftand right-handed technifermions, and $f$ is the PNGB decay constant. In particular, the anomaly factor relevant for $Z \rightarrow \gamma P^{a}$ is

$$
\mathcal{A}_{Z \gamma}=\frac{1}{2} \operatorname{Tr}\left[T^{a}\left(T_{3 L}+T_{3 R}-2 Q \sin ^{2} \theta_{W}\right) Q\right]
$$

so that if right handed technifermions are weak singlets, a PNGB with custodial isospin 0 $(1,2)$ has $\mathcal{A}_{\gamma Z}$ proportional to $\sin ^{2} \theta_{W}$ or $1-2 \sin ^{2} \theta_{W}\left(1-4 \sin ^{2} \theta_{W}, 1-2 \sin ^{2} \theta_{W}\right)$ [1].

The $Z$-boson decays to $\gamma P^{a}$ with width [1]

$$
\Gamma_{Z \rightarrow \gamma P^{a}}=2.3 \times 10^{-5} \mathrm{GeV}\left(\frac{123}{f}\right)^{2}\left(N_{T C} \mathcal{A}_{Z \gamma}\right)^{2}\left(\frac{M_{Z}^{2}-M_{P}^{2}}{M_{Z}^{2}}\right)^{3}
$$

where $M_{Z}$ and $M_{P}$ are the $Z$ and scalar masses, It is reasonable to expect a branching ratio of order $10^{-5}$, which is large enough to be visible in current LEP experiments.

Once produced, the PNGB has many possible decay paths [2]:

- In models where $P^{a}$ only decays to electroweak gauge bosons, the dominant mode is a two-photon decay.

- Another possibility is that the PNGB mainly decays into particles in an invisible sector. In this case, the event contains a single hard photon and missing energy.

- If $P^{a}$ gets its mass from effective four-fermion couplings due to extended technicolor interactions, then it could decay to a fermion/anti-fermion pair via twotechnifermion/two-fermion couplings. In many models, the coupling between $P^{a}$ and the fermions is proportional to the fermion mass [9].

- If some technifermions are colored, a color-neutral PNGB can decay to a pair of gluons through a triangle diagram with internal technifermions.

- Any PNGB produced by the process $Z \rightarrow \gamma P^{a}$ can decay through a photon and offshell $Z$. This three-body mode is significant only when no two-body decay paths are open [2]. For this reason, and because there is no data suggestive of this decay path [10], we do not consider this mode further.

As we examine the LEP data for signs that PNGBs are being produced at the $Z$ pole, we will study the final states that correspond to each of the two-body decay modes. In this way, we will cover a range of scenarios for the dominant decay modes of the PNGB. 


\section{Final State Kinematics}

To search the data for evidence of a light PNGB, we must determine how the experimental cuts would affect the size of the signal. Hence, we need to find the energies and directions of the various final state particles. We can divide the decay process into two steps: the two-body decay $Z \rightarrow P^{a} \gamma$ and the subsequent decay of $P^{a}$.

However $P^{a}$ decays, the final state at LEP always contains one photon of energy

$$
E_{\gamma}=\frac{M_{Z}^{2}-M_{P}^{2}}{2 M_{Z}}
$$

For example, if $M_{P}$ is less than $65 \mathrm{GeV}$ then $E_{\gamma}$ always exceeds $20 \mathrm{GeV}$. If the $P^{a}$ decays invisibly, no further kinematic information is required.

If the $P^{a}$ decays to pairs of photons, gluons, or fermions, we will need the energies and directions of the decay products. We will consider $P^{a} \rightarrow b \bar{b}$ explicitly. The results may be applied to other final states by substituting the appropriate mass for $m_{b}$ in the final formulae.

Starting in the $P^{a}$ rest frame, where the b-quarks are produced back-to-back, the four-momenta for the $b$ and $\bar{b}$ quarks are, respectively,

$$
\begin{aligned}
& \mathcal{P}_{b}=\left(\frac{1}{2} M_{P}, \mathrm{p}_{2} \cos \theta, \mathrm{p}_{2} \sin \theta \cos \omega, \mathrm{p}_{2} \sin \theta \sin \omega\right) \\
& \mathcal{P}_{\bar{b}}=\left(\frac{1}{2} M_{P},-\mathrm{p}_{2} \cos \theta,-\mathrm{p}_{2} \sin \theta \cos \omega,-\mathrm{p}_{2} \sin \theta \sin \omega\right),
\end{aligned}
$$

where

$$
\mathrm{p}_{2}=\sqrt{\frac{1}{4} M_{P}^{2}-M_{b}^{2}}
$$

while $\theta$ and $\omega$ are defined such that if the $P^{a}$ boost is in the $(1,0,0)$ direction, the b-quark moves along $(\cos \theta, \sin \theta \cos \omega, \sin \theta, \sin \omega)$.

Boosting to the lab frame with the beamline in the $\hat{x}$ direction and the $P^{a}$ threemomentum pointing along $(\cos \alpha, \sin \alpha \cos \beta, \sin \alpha, \sin \beta)$, we get:

$$
\begin{aligned}
& \mathrm{E}_{b}=\frac{M_{Z}}{4}[(1+\sqrt{(1-y)} \cos \theta)+x(1-\sqrt{(1-y)} \cos \theta)] \\
& \mathrm{E}_{\bar{b}}=\frac{M_{Z}}{4}[(1-\sqrt{(1-y)} \cos \theta)+x(1+\sqrt{(1-y)} \cos \theta)]
\end{aligned}
$$

where $y=4 M_{b}^{2} / M_{P}^{2}$ and $x=M_{P}^{2} / M_{Z}^{2}$. 
To apply experimental cuts to our signal, we will need the direction cosines of the particle momenta and the angles of separation between the various particles. The direction cosine between the beamline and the b-quark is

$$
\cos \mathcal{X}_{2}=\frac{[(1+\sqrt{(1-y)} \cos \theta)-x(1-\sqrt{(1-y)} \cos \theta)] \cos \alpha-2 \sqrt{x} \sin \theta \cos \omega \sin \alpha}{\sqrt{[(1+\sqrt{(1-y)} \cos \theta)-x(1-\sqrt{(1-y)} \cos \theta)]^{2}+4 x \sin ^{2} \theta}}
$$

The direction cosine $\cos \mathcal{X}_{3}$ for the $\bar{b}$-quark is obtained by reversing the sign of each $\cos \theta$ and $\sin \theta$ in equation (3.5). The direction cosine between the photon and the b-quark is

$$
\cos \mathcal{X}_{12}=\frac{-[(1+\sqrt{(1-y)} \cos \theta)-x(1-\sqrt{(1-y)} \cos \theta)]}{\sqrt{[(1+\sqrt{(1-y)} \cos \theta)-x(1-\sqrt{(1-y)} \cos \theta)]^{2}+4 x \sin ^{2} \theta}}
$$

The direction cosine $\cos \mathcal{X}_{13}$ between the photon and the anti-b-quark follows by sending $\cos \theta \rightarrow-\cos \theta$ in equation (3.6). The angle between the quark and anti-quark is

$$
\mathcal{X}_{23}=2 \pi-\left(\mathcal{X}_{12}+\mathcal{X}_{13}\right)
$$

since the event is planar.

\section{4. $Z \rightarrow \gamma P^{a} \rightarrow \gamma \gamma \gamma$}

The three photon events should yield a distinctive signal, including a hard photon whose energy is fixed by $M_{P}$. We used a Monte Carlo integration over $\theta, \alpha$ and $\omega$ to calculate the effects of the DELPHI experimental cuts 4 on the signal:

- The energy of each photon is greater than $10 \mathrm{GeV}$. This eliminates the signal for $P^{a}$ heavier than $80 \mathrm{GeV}$.

- All three photons in a given event should have polar angles between $20^{\circ}$ and $160^{\circ}$; two of the three should lie in the restricted range $42^{\circ}<\theta<$ $138^{\circ}$. This uniformly removes about $20 \%$ of the signal.

- The most energetic photon should carry energy greater than $20 \mathrm{GeV}$. This has no effect on the number of signal events.

- The most energetic photon should have polar angle between $40^{\circ}$ and $140^{\circ}$. Like the second cut, this one removes $20 \%$ of the signal.

- The angular separations between the least energetic photon and each of the other two should be greater than $20^{\circ}$. This cut mainly affects light $P^{a}$ 
which are produced with large momenta and therefore decay into two photons with small angular separation.

In addition, the DELPHI experiment [4 required the absence of vertex detector tracks pointing to the clusters in the electromagnetic calorimeter ('photons') and the general absence of energetic tracks coming from the beam-crossing point.

Depending on the PNGB mass, between $40 \%$ and $75 \%$ of the signal survives the cuts on the photons, as shown in fig. 1.

The size of the expected signal may now be compared with the experimental upper limit [4] on the branching ratio: B.R. $(Z \rightarrow \gamma \gamma \gamma)<1.7 \times 10^{-5}$. We set the PNGB decay constant to $f=123 \mathrm{GeV}$, and used (2.4) to find the maximum value of $\left[N_{T C} \mathcal{A}_{Z \gamma}\right]$ as a function of $M_{P}$, as shown in fig. 2. The limit is of order 2-3 for masses below about 60 $\mathrm{GeV}$, and weakens with increasing mass. The lowest mass for which the bounds apply is set by the angular separation cut; the highest mass, by the $10 \mathrm{GeV}$ photon energy cut.

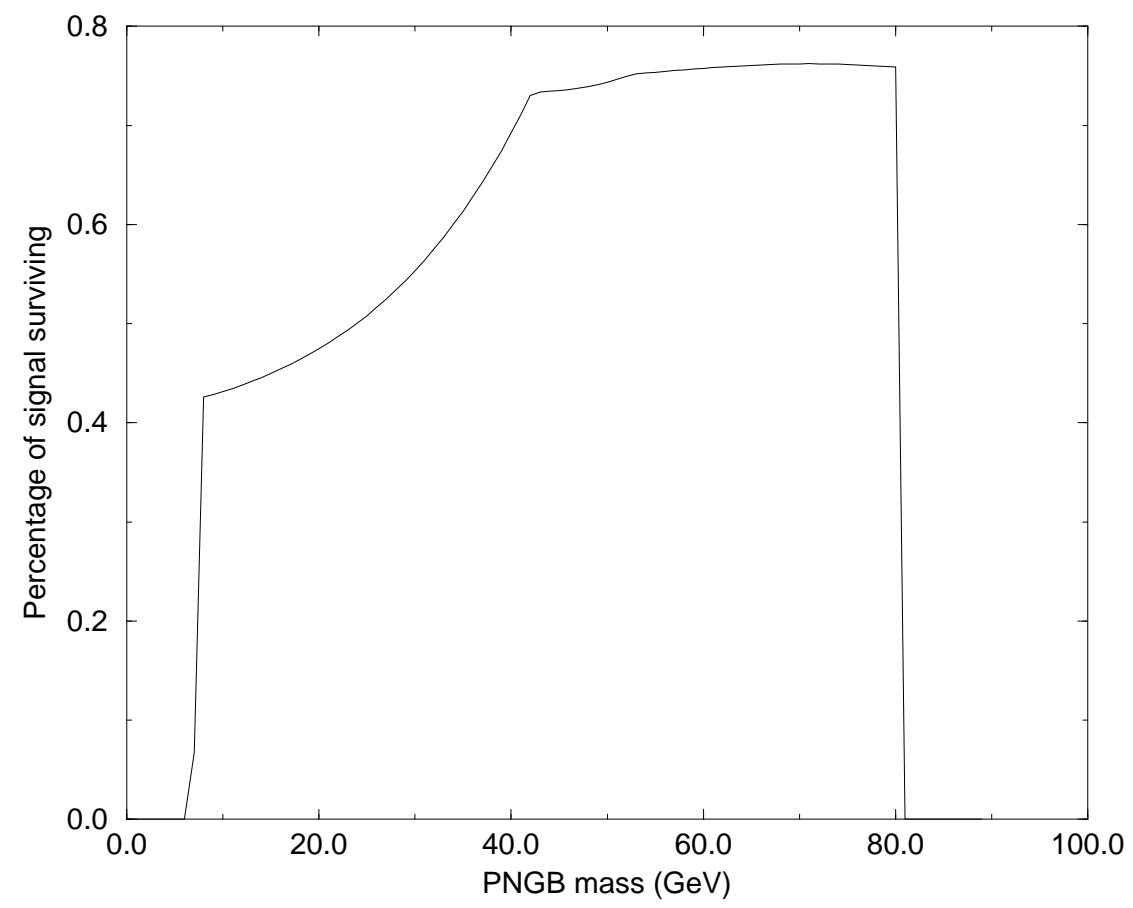

Fig. 1. Percentage of $Z \rightarrow \gamma P^{a} \rightarrow \gamma \gamma \gamma$ signal surviving cuts used by the DELPHI experiment, as a function of PNGB mass.

The left boundary comes from the angular separation cut; the shape at low mass and the right-hand boundary, from the $10 \mathrm{GeV}$ photon energy cut. 


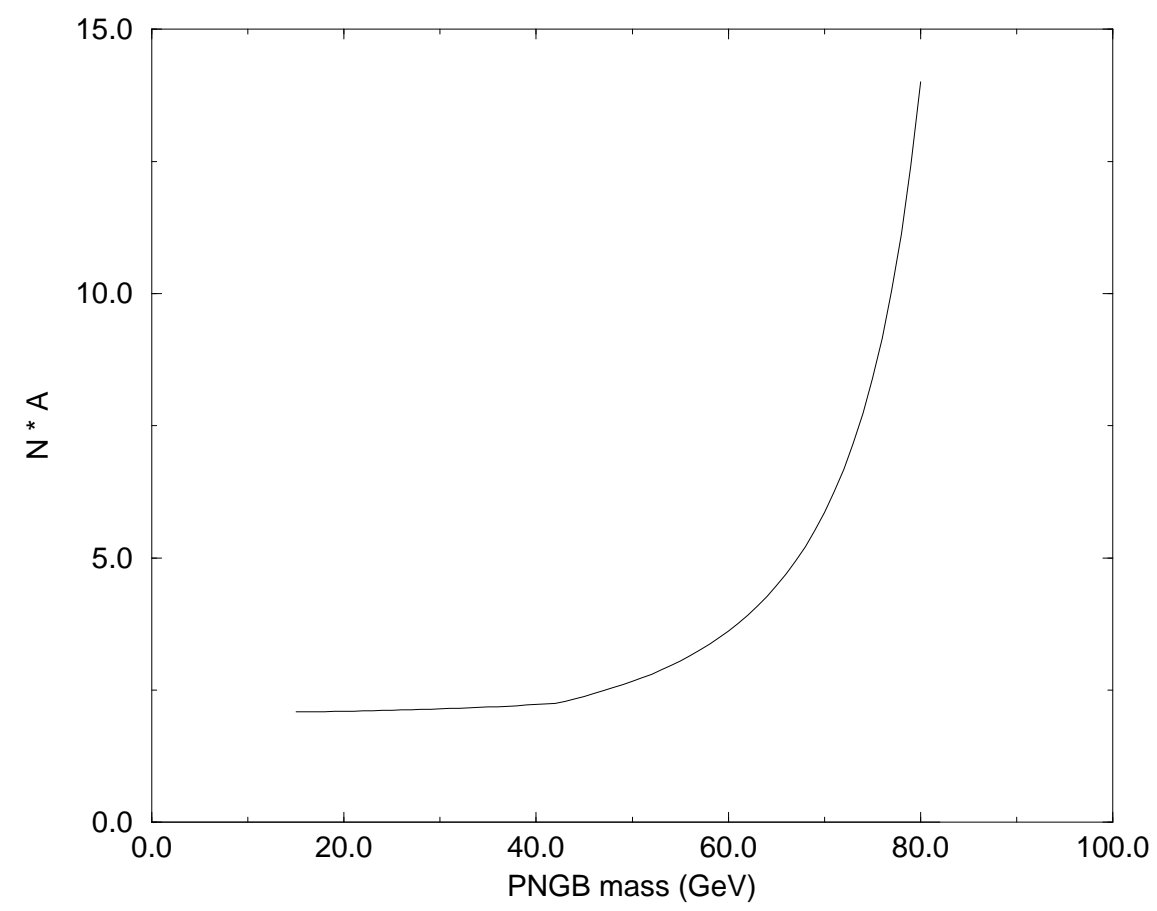

Fig. 2. Upper bound (at $95 \%$ c.l.) on $N_{T C} \mathcal{A}_{Z \gamma}$ as a function of $P^{a}$ mass in models where $P^{a}$ decays dominantly to photons and $f=123 \mathrm{GeV}$.

Derived from eq. (2.5) and DELPHI data on $Z \rightarrow \gamma \gamma \gamma$.

5. $Z \rightarrow \gamma P^{a} \rightarrow \gamma+\not$

If the scalar's dominant decay is invisible, only the photon from the $Z$ decay will be directly observeable. The experimental candidate events are characterized by one energetic photon in an otherwise empty detector: no tracks, muon hits, or other energy deposits (except as consistent with noise). To suppress background to new physics, cuts are also placed on the single photon. To assess the effects of these on the signal, we performed a Monte Carlo integration over the variable $\theta$, imposing the following conditions [5] used by the L3 Collaboration:

- The energy of the photon should be greater than $15 \mathrm{GeV}$. This eliminates the signal for PNGB's heavier than $75 \mathrm{GeV}$.

- The photon must have a polar angle between $20^{\circ}$ and $160^{\circ}$ (excluding the regions $34.5^{\circ}-44.5^{\circ}$ and $135.5^{\circ}-145.5^{\circ}$ ). Since the $Z$ decay to scalar plus photon is isotropic, this cut uniformly reduces the signal by approximately one third.

The L3 paper [5] plots the number of events as a function of photon energy, using energy bins $2 \mathrm{GeV}$ wide. No bin shows an excess of more than one event over expected 
standard model backgrounds from $\nu \bar{\nu} \gamma, e^{+} e^{-} \gamma$ and $\gamma \gamma \gamma$ final states. Assuming $f=123$ $\mathrm{GeV}$ and applying Poisson statistics, we find at $95 \%$ c.l. that $\left[N_{T C} \mathcal{A}_{Z \gamma}\right] \lesssim 1.5$ for $40<$ $M_{P}<70 \mathrm{GeV}$. The precise limit as a function of $M_{P}$ is shown in Figure 3.

We performed a similar analysis based on the OPAL data [6]. In this experiment, single-photon events were selected if the photon carried energy above $1.75 \mathrm{GeV}$ and restricted to the polar region $|\cos \alpha|<0.7$. OPAL reports [6] an upper bound (at 95\% c.l.) of $4.3 \times 10^{-6}$ on the branching ratio for radiative decay of the $Z$ to an invisibly-decaying scalar particle $X$ with mass less than $64 \mathrm{GeV}$, and an upper bound of $1.4 \times 10^{-5}$ for a mass less than $84 \mathrm{GeV}$. This sets the approximate limit $\left[N_{T C} \mathcal{A}_{Z \gamma}\right]<1$ for PNGB masses less than $40 \mathrm{GeV}$. As shown in fig. 3, the bounds from the OPAL data are weaker than those from L3 for values of $M_{P}$ accessible to both experiments.

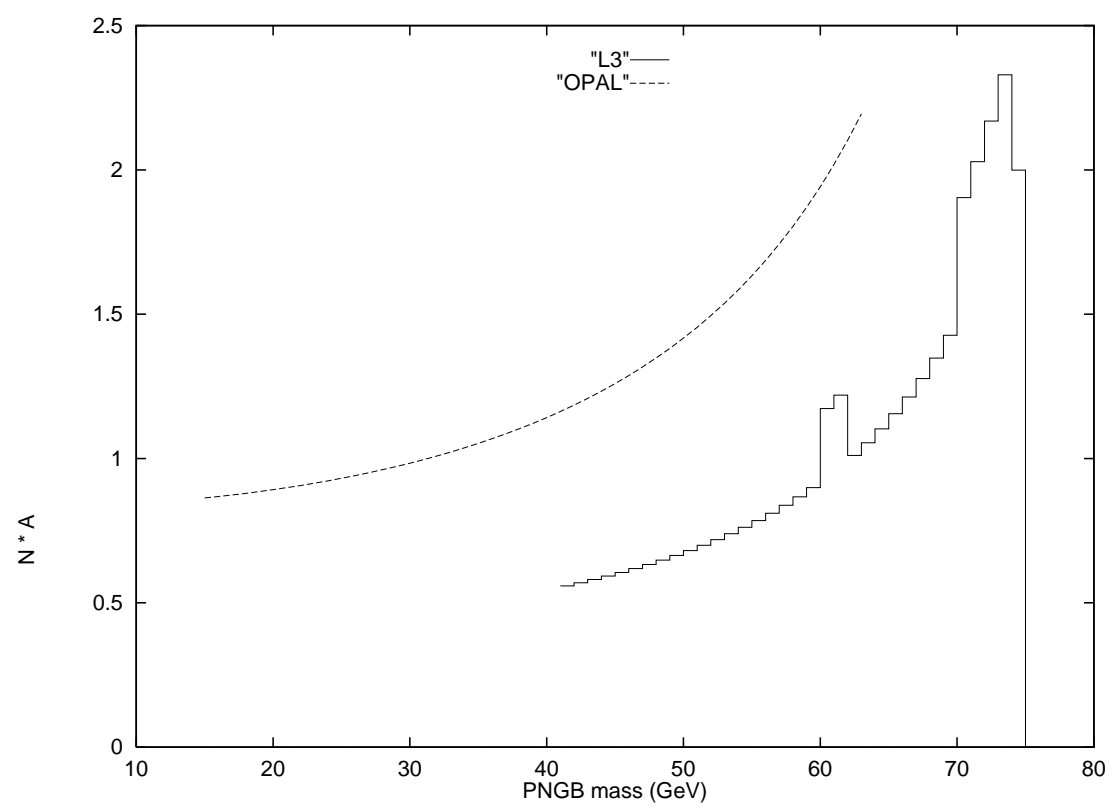

Figure 3. Upper bound (at $95 \%$ c.l.) on $N_{T C} \mathcal{A}_{Z \gamma}$ as a function. of PNGB mass in models where PNGB decays invisibly and $f=123 \mathrm{GeV}$. Derived from eq. (2.5) and L3 and OPAL data on $Z \rightarrow \gamma+\not$.

6. $Z \rightarrow \gamma P^{a} \rightarrow \gamma$ jet jet

If $P^{a}$ can decay into gluons, this mode dominates. Otherwise, if the PNGB couples directly to ordinary fermions in proportion to the fermion masses, then $P^{a} \rightarrow b \bar{b}$ is expected to be the most important mode, with decays to $c \bar{c}$ and $\tau^{+} \tau^{-}$taking over for $M_{P}<2 m_{b}$. In either case, the L3 data on isolated hard photons in hadronic $Z$ decays [7], can be 
used to search for $P^{a}$. We explicitly discuss the case in which fermionic decays of $P^{a}$ predominate. The limits on models in which $P^{a} \rightarrow g g$ dominates are nearly identical since no flavor tagging is employed in the selection cuts and since the jet-jet angular separation cut essentially eliminates most $P^{a}$ lighter than $2 m_{b}$.

Following the L3 experimental analysis [7], we applied the following cuts to our signal:

- The photon energy is greater than $5 \mathrm{GeV}$. This suppresses the signal for $P^{a}$ heavier than $85 \mathrm{GeV}$.

- The photon should lie between polar angle $45^{\circ}$ and $135^{\circ}$. This uniformly reduces the signal strength by about $30 \%$.

- The hadronic jets should lie in the region $5^{\circ}<\theta<175^{\circ}$. This reduces the signal by another few percent.

- The jets should be separated by at least $20^{\circ}$ from one another and from the photon. The main effect is to suppress the signal for low scalar masses.

About $70 \%$ of the signal survives these cuts for $P^{a}$ masses between 8 and $85 \mathrm{GeV}$.

The L3 collaboration [7] reports a limit on radiative $Z$ decay to a hadronically decaying narrow resonance, $Y$, that may be approximately written as

$$
\sigma\left(e^{+} e^{-} \rightarrow Z \rightarrow \gamma Y\right) \cdot \text { B.R. }(Y \rightarrow \text { hadrons }) \lesssim 5 \mathrm{pb}, \quad \text { for } M_{Y} \lesssim 85 \mathrm{GeV}
$$

Comparison with our expected signal for a hadronically decaying $P^{a}$ implies $\left[N_{T C} \mathcal{A}_{Z \gamma}\right] \leq 5$ for $M_{P} \lesssim 50 \mathrm{GeV}$ and $f=123 \mathrm{GeV}$. The bound loosens to $\left[N_{T C} \mathcal{A}_{Z \gamma}\right] \leq 10$ for $M_{P} \approx 60$ $\mathrm{GeV}$ and becomes even weaker for heavier $P^{a}$ because the production rate declines so steeply with increasing $M_{P}$.

\section{Applications to Technicolor Models}

Having reported the bounds that LEP sets on neutral PNGB in the general form of limits on $\left[N_{T C} \mathcal{A}_{Z \gamma}\right]$, we now apply them to several technicolor scenarios. This allows us to see what classes of models are most strongly constrained now and which will be probed by more stringent experimental limits on rare $Z$ decays.

The benchmark technicolor model which includes PNGB is one-family technicolor [11]. In this model, the technipion decay constant is $f=v / 2=123 \mathrm{GeV}$, and the neutral PNGB can be described in terms of technifermion quantum numbers as

$$
P^{1} \sim\left(\bar{Q} \gamma_{5} Q-3 \bar{L} \gamma_{5} L\right), \quad P^{3} \sim\left(\bar{Q} \gamma_{5} \tau_{3} Q-3 \bar{L} \gamma_{5} \tau_{3} L\right)
$$


where the superscript on $P$ indicates the dimension of the custodial isospin representation. These can decay to two jets either through direct ETC couplings to quarks or through the coupling of gluons to the techniquarks. The corresponding anomaly factors are

$$
\mathcal{A}_{Z \gamma}^{1}=\frac{1}{3 \sqrt{3}} s_{W}^{2} \approx 0.044, \quad \mathcal{A}_{Z \gamma}^{3}=\frac{1}{\sqrt{3}}\left(\frac{1}{4}-s_{W}^{2}\right) \approx 0.012 .
$$

Note that, as mentioned in section 2, the state with $\mathrm{I}=1$ has an anomaly factor suppressed by $\left(\frac{1}{4}-s_{W}^{2}\right)$. Both anomaly factors are so small that the LEP limits on radiative hadronic $Z$ decays set no useful bounds on one-family technicolor.

Since $\Gamma(Z \rightarrow \gamma P)$ goes as $\left(N_{T C} \mathcal{A}_{Z \gamma} / f\right)^{2}$, models more likely to be probed by the LEP data will need to have smaller $f$ or larger $\mathcal{A}_{Z \gamma}$ than one-family technicolor. Several models with small technipion decay constants have recently been proposed; we consider these first.

In ref. [12], the authors introduce one-family technicolor models in which the QCD interactions combine with near-critical ETC interactions to enhance the techniquark masses relative to those of the technileptons. The main attraction of such models is that nondegenerate technifermions can potentially lead to a small value of the electroweak radiative correction parameter, $S$ [13]. The side-effect of interest here is that the technipions composed of technineutrinos and technileptons can be relatively light - with masses as low as 50-100 GeV - and have small decay constants (of order $40 \mathrm{GeV}$ ). Due the splitting between the techniquarks and technileptons, the model has a smaller chiral flavor symmetry than one-family technicolor, and the neutral PNGB are

$$
P_{Q}^{3} \sim \bar{Q} \gamma_{5} \tau^{3} Q, \quad P_{L}^{3} \sim \bar{L} \gamma_{5} \tau^{3} L, \quad P^{1} \sim \bar{Q} \gamma_{5} Q-3 \bar{L} \gamma_{5} L
$$

The NGB that is eaten by the $Z$ is largely composed of techniquarks $\left(f_{Q}>>f_{L}\right)$ and the remaining PNGB mass eigenstates are approximately (in the limit of large isospin breaking)

$$
P_{N} \sim \bar{N} \gamma_{5} N, \quad P_{E} \sim \bar{E} \gamma_{5} E
$$

The lighter of these, $P_{N}$, has $\mathcal{A}_{Z \gamma}=0$ since the technineutrino carries no electric charge. The heavier $P_{E}$ can still be light enough to be produced at LEP. Although $P_{E}$ has an $I=0$ component, the terms in its anomaly factor proportional to $-2 s_{W}^{2}$ and to $1-2 s_{W}^{2}$ have identical coefficients, so the full anomaly factor is

$$
\mathcal{A}_{Z \gamma}^{E}=\frac{1}{\sqrt{2}}\left(\frac{1}{4}-s_{W}^{2}\right)
$$


Even the reduced $f_{L}$ in this model is not sufficient to overcome this. In consequence, $P_{E}$ will not be visible at LEP no matter how it decays.

The multiscale TC models introduced in [14] include technifermions in large technicolor representations and can also have light neutral PNGB with small decay constants [14] [15]. While the lightest PNGB cant have a mass below $M_{Z}$, this PNGB, however, is largely composed of the $\mathrm{I}=1$ state $P_{L}^{3} \sim \bar{L} \gamma_{5} \tau_{3} L$ so that although $f_{L} \sim 30 \mathrm{GeV}$, the anomaly factor is suppressed by $\left(\frac{1}{4}-s_{W}^{2}\right)$. In the most optimistic case, in which the PNGB decays half the time to a pair of photons [17], the model with $N_{T C}=6$ gives a signal 10 times smaller than the LEP bound. If the decays are primarily hadronic (through ETC interactions), the signal lies even further below the relevant limit. P.

Clearly, small $f$ is not enough: we also need models with large $\mathcal{A}_{Z \gamma}$, e.g., those in which the lightest technifermions belong to larger representations of $S U(2)_{W}$. Consider, for example, a scenario suggested in [1] in which the left-handed technifermions are a weak isotriplet of techniquarks, Q, with hypercharge $y$ and one of technileptons, L, with hypercharge $-3 y$, while the right-handed technifermions are weak singlets. In this case, $f=v / 4$ and there are $\mathrm{I}=0$ and $\mathrm{I}=2$ states

$$
P^{1} \sim\left(\bar{Q} \gamma_{5} Q-3 \bar{L} \gamma_{5} L\right), \quad P_{+}^{5} \sim\left(\bar{Q} \gamma_{5} T^{8} Q+\bar{L} \gamma_{5} T^{8} L\right)
$$

where $T^{8}=\operatorname{diag}(1,-2,1)$, with sizeable anomaly factors:

$$
\mathcal{A}_{Z \gamma}^{1}=6 \sqrt{2} s_{W}^{2} y^{2}, \quad \mathcal{A}_{Z \gamma}^{5}=\frac{1}{\sqrt{3}}\left(1-2 s_{W}^{2}\right) .
$$

No matter what the (model-dependent) dominant decays of these technipions, the LEP data place strong limits on the size of $N_{T C}$ as a function of $M_{P}$.

If the PNGB decay invisibly, with $f=v / 4$ the experimental limit is (cf. section 5)

$$
2 N_{T C} \mathcal{A}_{Z \gamma} \leq 1 \text { (2) when } \quad M_{P} \lesssim 65 \mathrm{GeV}(75 \mathrm{GeV})
$$

If the $I=2$ state is sufficiently light, then (7.8) combined with (7.7) yields

$$
N_{T C} \leq 1 \text { (3) when } M_{P_{+}^{5}} \lesssim 65 \mathrm{GeV}(75 \mathrm{GeV})
$$

so that LEP has excluded all such models with $M_{P_{+}^{5}} \lesssim 65 \mathrm{GeV}$ and allows only $N_{T C}=2,3$ if $65 \lesssim M_{P_{+}^{5}} \lesssim 75 \mathrm{GeV}$. If only the $\mathrm{I}=0$ state is light enough to be produced at LEP, then

\footnotetext{
4 Based on the results of [17] and lowering the scaling factor, $\kappa$, to 1.0 [14].
} 
for techniquark hypercharge $y>1 / 3$, the limits are equivalent to those shown above. The non-observation of these PNGB therefore implies that either $P^{1}$ and $P_{+}^{5}$ are heavy or $y$ is small.

If $\gamma \gamma$ decays dominate, the LEP data tell us (cf. section 4)

$$
2 N_{T C} \mathcal{A}_{Z \gamma} \leq 2.5 \text { (5) for } \quad M_{P} \lesssim 50 \mathrm{GeV}(70 \mathrm{GeV})
$$

This excludes models in which $P^{1}$ has a mass less than $50 \mathrm{GeV}$ and the techniquark hypercharge exceeds $\frac{1}{2}$. Models in which $P_{+}^{5}$ is light or the techniquark hypercharge is smaller are allowed within the following constraints

$$
\begin{array}{lll}
N_{T C} \leq 4 & \text { for } & M_{P_{+}^{5}} \lesssim 50 \mathrm{GeV} \\
N_{T C} \leq 5 & \text { for } & M_{P^{1}} \lesssim 50 \mathrm{GeV} \text { and } y \geq \frac{1}{3}
\end{array}
$$

The extension to heavier PNGB and smaller $y$ follows from (7.7) and (7.10).

Finally, if hadronic decays of $P^{1}$ or $P_{+}^{5}$ dominate, the data (6.1) place an upper bound of $5 \mathrm{pb}$ on the quantity $\left[70 \% \cdot \sigma_{\text {tot }}^{Z} \cdot\right.$ B.R. $\left.\left(Z \rightarrow \gamma P^{a}\right)\right]$ where the B.R. comes from (2.4) and the factor of $70 \%$ is the roughly mass-independent acceptance of the cuts. In the range $20<M_{P}<60 \mathrm{GeV}$, this quantity is approximately linear in $M_{P}$ :

$$
\frac{1}{4}\left[5.5+2.2\left(1-\frac{M_{P}}{20 \mathrm{GeV}}\right)\right] \mathrm{pb} \cdot\left(N_{T C} \mathcal{A}_{Z \gamma}\right)^{2}
$$

where $f=v / 4$. Eq. (7.12) does not set a strong lower limit on the mass of $P_{+}^{5}$; even if $M_{P}{ }^{5}$ is as small as $20 \mathrm{GeV}, N_{T C}=6$ is allowed. On the other hand, for $y=1$, the $P^{1}$ must weigh at least $60 \mathrm{GeV}$; if $y=\frac{1}{2}$, then $M_{P^{1}}>40 \mathrm{GeV}$ unless $N_{T C} \leq 5$.

\section{Summary}

LEP data on rare $Z$ decays are beginning to set limits on the presence of the light neutral pseudo Nambu-Goldstone bosons characteristic of non-minimal technicolor models. Not surprisingly, the data most strongly constrains models with a large $Z \gamma P^{a}$ coupling. Probing multiscale technicolor models which have a smaller $Z \gamma P^{a}$ coupling but also have small technipion decay constants will be possible with an improvement of order a factor of 10 in the LEP bounds on rare $Z$ decays. 


\section{Acknowledgments}

The authors thank Bing Zhou for experimental information. We also thank Sekhar Chivukula and Ken Lane for comments on the manuscript. EHS acknowledges the support of an NSF Faculty Early Career Development (CAREER) Award and the hospitality of the Aspen Center for Physics where this work was completed. This work was supported in part by the National Science Foundation under grant PHY-9501249 and by the U.S. Department of Energy under grant DE-FG02-91ER40676. 


\section{References}

[1] A. Manohar and L. Randall, Phys. Lett. B246 (1990) 537

[2] L. Randall and E.H. Simmons, Nucl. Phys. B380 (1992) 3

[3] V. Lubicz, Nucl. Phys. B404 (1993) 559

[4] DELPHI Collaboration, P. Abreu et al., Phys. Lett. B327 (1994) 386

[5] L3 Collaboration, Measurement of Energetic Single-Photon Production at LEP, CERN-PPE/94-216 (1994)

[6] OPAL Collaboration, Measurement of Single Photon Production in $e^{+} e^{-}$Collisions near the $Z^{0}$ Resonance, CERN-PPE/94-105 (1994)

[7] L3 Collaboration, O. Adriani et al., Phys. Lett. B292 (1992) 472

[8] S. Dimopoulos, S. Raby and G.L. Kane, Nucl. Phys. B182 (1981) 77 ;

J. Ellis et al., Nucl. Phys. B182 (1981) 529 ;

B. Holdom, Phys. Rev. D24 (1981) 1441

[9] S. Dimopoulos and L. Susskind, Nucl. Phys. B155 (1979) 237 ;

E.Eichten and K. Lane, Phys. Lett. B90 (1980) 125

[10] See, e.g., L. Randall and N. Rius, Phys. Lett. B309 (1993) 365

[11] E. Farhi and L. Susskind, Phys. Rep. 74 (1981) 277

[12] T. Appelquist and J. Terning, Phys. Lett. B315 (1993) 139

[13] M. Golden and L. Randall, Nucl. Phys. B361 (1991) 3 ;

M. E. Peskin and T. Takeuchi, Phys. Rev. Lett. 65 (1990) 964 ;

B. Holdom and J. Terning, Phys. Lett. B247 (1990) 88 ;

W. J. Marciano and J. L. Rosner, Phys. Rev. Lett. 65 (1990) 2963 ;

D. Kennedy and P. Langacker, Phys. Rev. Lett. 65 (1990) 2967; Phys. Rev. D44 (1991) 1591

[14] K. Lane and E. Eichten, Phys. Lett. B222 (1989) 274

[15] K. Lane and M.V. Ramana, Phys. Rev. D44 (1991) 2678

[16] K. Lane, Color-Singlet Technipions at the Tevatron, BUHEP-95-23, hep-ph9507289

[17] V. Lubicz and P. Santorelli, Production of Neutral Pseudo-Goldstone bosons at LEPII and NLC in multiscale walking technicolor models, BUHEP-95-16, DSF 21/95, hep$\mathrm{ph} / 9505336$ 